\title{
Redox-Induced Gating of the Exchange Interactions in a Single Organic Diradical
}

Rocco Gaudenzi, ${ }^{\dagger}$ Joeri de Bruijckere, ${ }^{\dagger}$ Daniel Reta, ${ }^{\ddagger}, I_{\odot}$ Ibério de P. R. Moreira, ${ }^{\ddagger}$ Concepció Rovira, ${ }^{\S}$ Jaume Veciana, ${ }^{\S}$ Herre S. J. van der Zant, ${ }^{\dagger}$ and Enrique Burzurí ${ }^{*}{ }^{\dagger}$

${ }^{\dagger}$ Kavli Institute of Nanoscience, Delft University of Technology, Lorentzweg 1, 2628 CJ Delft, The Netherlands

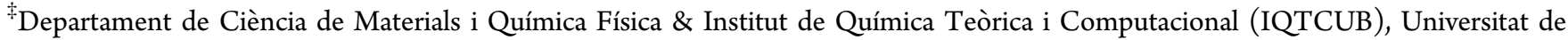
Barcelona, C/Martí i Franquès 1, 08028 Barcelona, Spain

${ }^{\S}$ Institut de Ciència de Materials de Barcelona (ICMAB-CSIC) and CIBER-BBN, Campus de la UAB, 08193, Bellaterra, Spain

\section{Supporting Information}

ABSTRACT: Embedding a magnetic electroactive molecule in a three-terminal junction allows for the fast and local electric field control of magnetic properties desirable in spintronic devices and quantum gates. Here, we provide an example of this control through the reversible and stable charging of a single all-organic neutral diradical molecule. By means of inelastic electron tunnel spectroscopy we show that the added electron occupies a molecular orbital distinct from those containing the two radical electrons, forming a spin system with three antiferromagnetically coupled spins. Changing the redox state of the molecule therefore switches on and off a parallel exchange path between the two radical spins through the added electron. This electrically controlled gating of the intramolecular magnetic interactions constitutes an essential ingredient of a single-molecule $\sqrt{\operatorname{SWAP}}$ quantum gate.

KEYWORDS: molecular electronics, organic radicals, quantum information, spintronics, diradicals
$\mathrm{F}$ ast, reversible, and local control of magnetic properties of molecular systems is sought after as a potential path for molecule-based spintronic devices ${ }^{1-3}$ and quantum information processing. ${ }^{4-6}$ The control of the intramolecular exchange coupling could allow, for instance, for the realization of a single-molecule quantum gate. ${ }^{7-10}$ One way to achieve such control at the single-molecule level is to embed a magnetic electroactive molecule in a solid-state junction and use the gate electrode to change its magnetic properties through a form of spin-electric coupling. ${ }^{71-17}$ Traditional candidates are singlemolecule magnets (SMMs), the magnetic parameters of which can be modulated with the addition of a charge ${ }^{18-20}$ or through magnetoelectric effects. ${ }^{21}$

A promising alternative to SMMs is offered by all-organic radical molecules, ${ }^{7}$ where the magnetism arises from the unpaired spins of carbon atoms. ${ }^{22,23}$ The simplicity of their spin structure and the absence of metal centers have proven to yield robust molecular junctions ${ }^{24-26}$ and potentially allow overcoming the limitations inherent to SMMs owing to low spinorbit coupling and hyperfine interaction. However, the existing experimental examples have shown either a relatively small electric control over the exchange coupling ${ }^{26}$ or a reduction of the molecule to a closed-shell system with no unpaired spins. ${ }^{27}$
Here, we report the reversible and stable reduction of a neutral diradical molecule in a three-terminal device, by means of a gate electrode. Inelastic electron tunneling spectroscopy (IETS) in the two stable redox states shows that the added electron magnetically couples to the two radical spins, preserving their open-shell character, while changing the magnetic state of the molecule from a singlet to a doublet state with three unpaired electrons. This ability to reversibly switch on the exchange couplings between the added electron and the two radical spins could form the base for a $\sqrt{\text { SWAP }}$ quantum gate. $7,28,29$

\section{RESULTS AND DISCUSSION}

The molecule we use is a neutral 2,4,6-hexakis(pentachlorophenyl)mesitylene diradical molecule, ${ }^{30}$ hereafter PTM-based diradical, schematically shown in Figure 1. It is made of three methyl carbon atoms connected via a central benzene ring. Two of these $\mathrm{C}$ atoms are methyl radicals with unpaired electrons, while the third binds a $\mathrm{H}$ that closes the

Received: March 6, 2017

Accepted: May 11, 2017

Published: May 11, 2017 


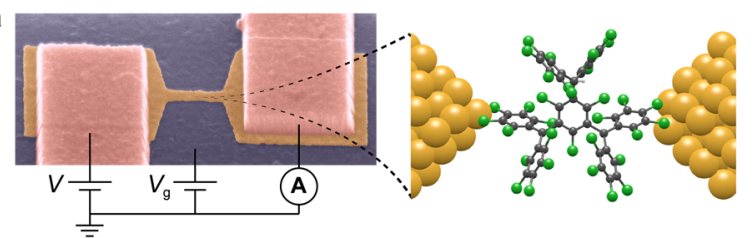

b
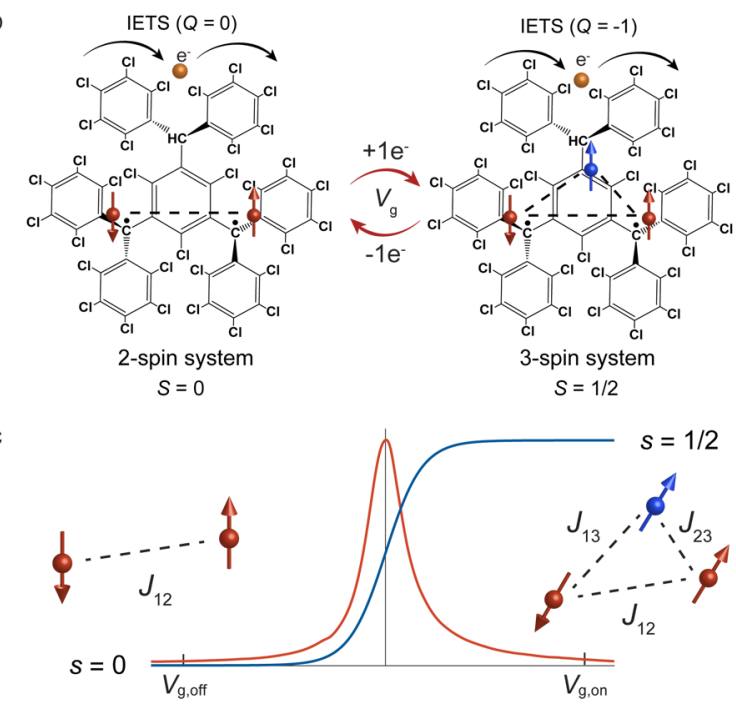

Figure 1. Organic diradical spin system. (a) Scanning electron microscope (SEM) false color image of a Au nanowire on top of an $\mathrm{Al}_{2} \mathrm{O}_{3} / \mathrm{AuPd}$ gate. (b) Structure and magnetism schematics of the neutral diradical and reduced form of the diradical. The red dots and the dashed lines mark the radical spins and the exchange interactions, respectively. A gate voltage allows reversibly adding a spin (blue dot) onto the redox center and, with that, switching on and off the magnetic couplings between the added electron and the two radical spins. For each state inelastic electron tunneling spectroscopy (IETS) is performed (yellow electron). (c) Differential conductance (red) and corresponding redox center spin value $s$ (blue) as a function of $V_{\mathrm{g}}$. Sweeping from $V_{\mathrm{g} \text {, off }}$ to $V_{\mathrm{g} \text {, on }}$, the site is progressively filled and $s$ increases from 0 to $1 / 2$. The value $V_{\mathrm{g} \text {, off }}\left(V_{\mathrm{g}, \text { on }}\right)$ marks the gate voltage at which the added spin stably resides off (on) the molecule.

electronic shell. The resulting molecule is a two-spin magnetic system. Two chlorinated phenyl rings attach and surround each methyl carbon in a propeller-like configuration, as seen in Figure 1. The single-molecule junction is formed when a single PTM-based diradical bridges the source and drain electrodes as illustrated in Figure 1a. The electric field produced by applying a gate voltage $\left(V_{\mathrm{g}}\right)$ to the third electrode is used to change the redox state of the molecule (Figure 1b). In transitioning between the two states, a high-conductance peak is traversed. On the right (left) of the peak, i.e., at $V_{\mathrm{g}, \text { on }}\left(V_{\mathrm{g}, \mathrm{off}}\right)$, the redox center has a stable spin $s=1 / 2(s=0)$. Additional details on the fabrication and molecule deposition can be found elsewhere ${ }^{31,32}$ and in the Methods.

We probe the excitation spectrum of an individual diradical molecule by measuring the dc current $I$ through the junction as a function of bias voltage $V$ and extracting the differential conductance $\mathrm{d} I / \mathrm{d} V$. Each step in the $\mathrm{d} I / \mathrm{d} V$ spectrum signals the opening of an inelastic electron current channel via the excited state of the molecule with energy $\mathrm{eV}$. Following the steps' energy as a function of magnetic field allows reading out the molecule's energy spectrum, providing a single-molecule analogue of electron-spin resonance spectroscopy. Figure 2a
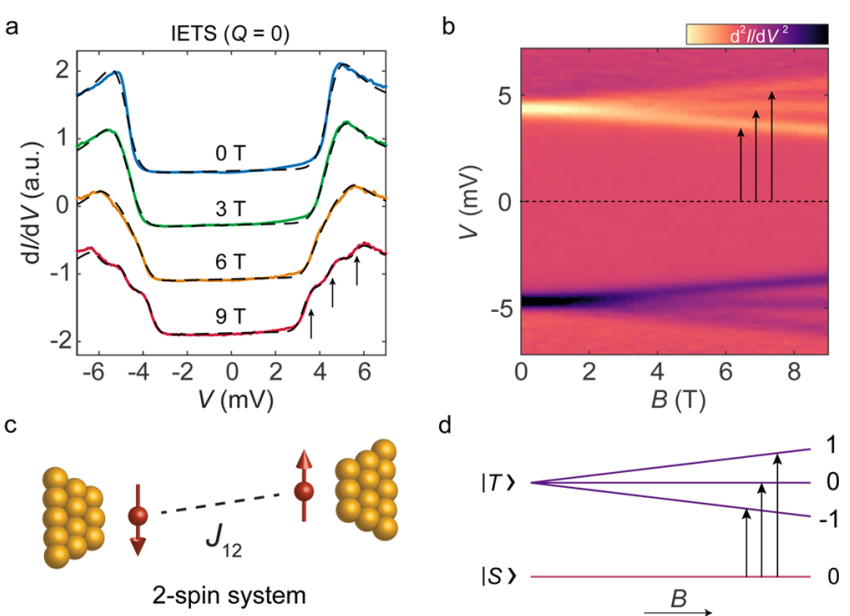

Figure 2. Magnetic spectrum of the neutral diradical. (a) Differential conductance $(\mathrm{d} I / \mathrm{d} V)$ spectra of the neutral diradical molecular junction at different magnetic fields and at a fixed gate voltage $V_{\mathrm{g}}=-2.3 \mathrm{~V}$. An excitation step at $\pm 4.65 \mathrm{mV}$ splits into three substeps under applied magnetic field. The superimposed dashed lines are fits using the model in ref 33. (b) $\mathrm{d}^{2} I / \mathrm{d} V^{2}$ color map showing the splitting as a function of $V$ and $B$. (c) Schematics of a two-spin system with exchange coupling $J_{12}$, confined between two gold electrodes. (d) Spin spectrum and allowed transitions for a two-spin system with antiferromagnetic $J_{12}$.

shows the $\mathrm{d} I / \mathrm{d} V$ spectra of a diradical junction at different magnetic fields $B$ at fixed gate voltage $V_{\mathrm{g}}=-2.3 \mathrm{~V}$. The spectrum taken at $0 \mathrm{~T}$ shows symmetric steps at $\pm 4.65 \mathrm{mV}$, which can be associated with transitions to excited spin states. The confirmation of the magnetic nature of the transitions is given by the evolution of the excitation energies as a function of the applied magnetic field $B$ (see Figure $2 \mathrm{~b}$, where the second derivative, $\mathrm{d}^{2} I / \mathrm{d} V^{2}$, is shown for clarity). The excitation step splits into three substeps as $B$ is increased. As shown in the level scheme of Figure $2 \mathrm{~d}$, this spectrum is consistent with the antiferromagnetically coupled two-spin system depicted in Figure $2 \mathrm{c}$ with an open-shell singlet $(S=0)$ ground state $|S\rangle$ and a triplet $(S=1)$ excited state $|T\rangle$.

We compare the experimental spectra with numerical simulations based on the tunneling model of ref 33 commonly used in scanning tunneling spectroscopy. The dashed black lines in Figure 2a show the results of these simulations. Within the framework of the model, we describe the diradical molecule by a model Hamiltonian with two spin- $1 / 2$ centers interacting through a Heisenberg exchange coupling $J_{12}$. For all magnetic field values, the data can be well fitted to this model with $J_{12}=$ $4.65 \mathrm{meV}$. The preference for the singlet ground state is ascribed to the distortion of the molecule in the solid-state device in analogy with previous studies on PTM-based neutral triradicals. ${ }^{26}$ We have verified the plausibility of this scenario by DFT calculations (see Supporting Information Section 2.2 for details).

A similar measurement is conducted at fixed $V_{\mathrm{g}}=3 \mathrm{~V}$. Figure 3a shows the resulting $\mathrm{d} I / \mathrm{d} V$ spectra for two different magnetic fields. At $B=0 \mathrm{~T}$, excitation steps appear at $V=-22,-19,+20$, and $+25 \mathrm{mV}$, together with a zero-bias peak ascribable to the Kondo effect. ${ }^{34,33}$ The asymmetry in bias-voltage positions with respect to $V=0$ and the different step heights can be respectively explained by a bias-dependent tuning of the exchange coupling and contributions from resonant transport with asymmetrically coupled electrodes. For increasing $B$, the 

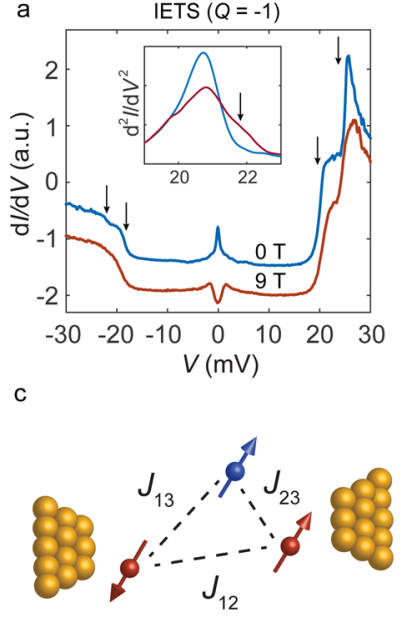

3-spin system

$$
\text { b }
$$
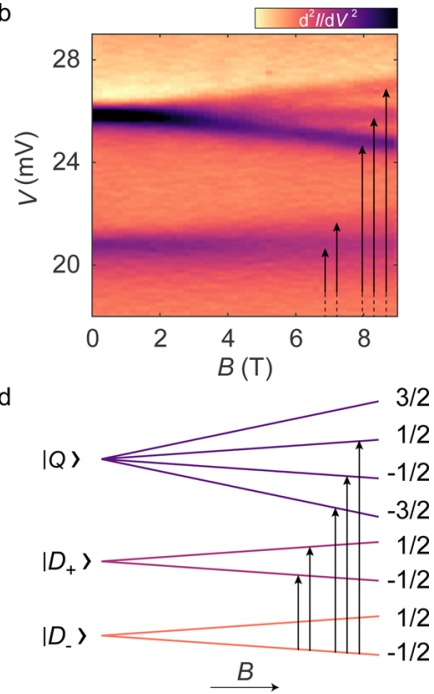

Figure 3. Magnetic spectrum of the reduced diradical. (a) Differential conductance $(\mathrm{d} I / \mathrm{d} V)$ spectra of the diradical junction at different magnetic fields and fixed $V_{\mathrm{g}}=+3 \mathrm{~V}$. Two excitation steps with energies +20 and $+25 \mathrm{mV}$ split into two and three, respectively, under the applied magnetic field. Inset: $\mathrm{d}^{2} I / \mathrm{d} V^{2}$ linecuts taken from (b) showing the splitting of the doublet $\left|D_{+}\right\rangle$. (b) $\mathrm{d}^{2} I / \mathrm{d} V^{2}$ color map as a function of $V$ and $B$. (c) Schematics of a three-spin system coupled via exchange interactions. The added electron, highlighted in blue, introduces two new exchange couplings, $J_{13}$ and $J_{23}$, with the intrinsic radical spins. (d) Spin spectra and allowed transitions for a three-spin system with antiferromagnetic $J_{12}$.

zero-bias peak evolves into a dip and the excitation steps split into two (Figure 3a, inset) and three for the low- and highenergy value, respectively. The $\mathrm{d}^{2} I / \mathrm{d} V^{2}$ color map of Figure $3 \mathrm{~b}$ shows this magnetic field evolution. From this set of excitations we deduce that the magnetic spectrum consists of a doublet ground-state multiplet $\left|D_{-}\right\rangle$, giving rise to the observed Kondo peak, a doublet excited multiplet $\left|D_{+}\right\rangle$, and a quartet excited multiplet $|Q\rangle$, as shown in Figure 3d (see Supporting Information Section 1 for more details). The excitations at +20 and $+25 \mathrm{mV}$ correspond therefore to the transitions $\left|D_{-}\right\rangle$ $\rightarrow\left|D_{+}\right\rangle$and $\left|D_{-}\right\rangle \rightarrow|Q\rangle$, respectively.

The spectrum we obtain at this gate voltage can be hosted only by a system like the one depicted in Figure 3c, where the electrostatically added electron occupies an empty orbital rather than either of the half-filled radical orbitals and couples to the two unpaired spins via the exchange interactions $J_{13}$ and $J_{23}$. This type of charging, observed also in two other molecular junctions of the 13 measured (see Methods for details on statistics), is in contrast to previously reported experiments on PTM monoradicals ${ }^{27}$ and other neutral diradical molecules. ${ }^{36}$ One of the possible explanations, explored by DFT calculations (see Supporting Information Section 2.3), is that the structural distortions determining the preference for the singlet ground state lead also to a concomitant reduction of the HOMOLUMO gap.

Differently from the neutral state, the excitation spectrum of the reduced state does not provide a unique solution for $J_{12}, J_{13}$, and $J_{23}$, but rather a subset of solutions in the space of the three exchange couplings (see Supporting Information Section 1). One scenario, obtained assuming that the coupling between the radical centers remains unchanged upon charging, yields for $J_{13}$ and $J_{23}$ the values 2 and $23 \mathrm{meV}$. In this scenario, the

asymmetry between $J_{13}$ and $J_{23}$ suggests that the added spin resides in the proximity of one of the radical centers. Gas-phase DFT calculations indicate that the added electron may be delocalized over the central phenyl ring (see Section 2.4 in the Supporting Information).

The transport characteristics of Figure 2 and Figure 3 are connected, as can be seen when varying the gate voltage $V_{\mathrm{g}}$ in a continuous way. Figure 4 a shows a $d I / d V$ map as a function of

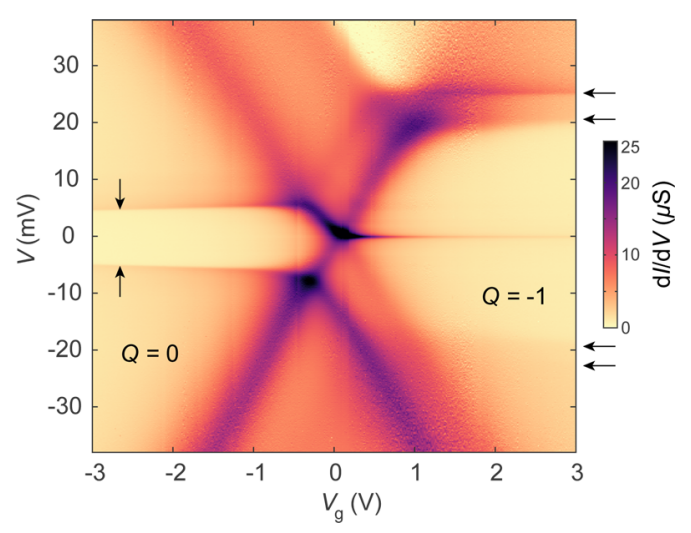

Figure 4. Exchange-coupling gating mechanism. (a) Differential conductance $(\mathrm{d} I / \mathrm{d} V)$ as a function of $V$ and $V_{\mathrm{g}}$ at $B=0 \mathrm{~T}$. Slanted high-conductance edges indicate resonant transport and separate two distinct, low-conductance regions where the charge state of the molecule is stable: a neutral charge state $(Q=0$, left $)$ and a reduced charge state $(Q=-1$, right). Excitations lines at $\pm 4.65 \mathrm{mV}$ (vertical arrows) are present in the $Q=0$ state. In the $Q=-1$ state excitation lines appear at $-19,-22,+20$, and $+25 \mathrm{mV}$ (horizontal arrows), along with a zero-bias line of enhanced $\mathrm{d} I / \mathrm{d} V$.

$V$ and $V_{\mathrm{g}}$. The high-conductance slanted edges crossing into a zero-bias peak at $V_{\mathrm{g}} \approx 0 \mathrm{~V}$ define a resonant electron transport region separating two areas of low conductance. These features signal the presence of a single molecule in the junction whose stable charge states, labeled by $Q=0$ and $Q=-1$, differ by one electron. The two lines in $Q=0$, marked by vertical arrows, correspond to the singlet-to-triplet excitation steps of Figure 2, while the arrows on the right-hand side indicate the excitations discussed in Figure 3.

The gate electrode thus provides a path to reversibly switch between the neutral and the reduced state of the diradical molecule. Along a horizontal path around zero bias, the highconductance peak of width $\Gamma \approx 5 \mathrm{meV}$ is traversed. In the proximity of the peak, the molecule is in a fully mixed-valence state: electrons from the electrodes are hopping on and off the redox center on a time scale $\tau=\hbar / \Gamma \approx 0.1$ ps. Upon application of a gate voltage $V_{\mathrm{g}}=+3 \mathrm{~V}\left(V_{\mathrm{g}}=-3 \mathrm{~V}\right)$, within a time $\tau$ the redox center acquires a discrete occupation number and a stable spin $s=1 / 2(s=0)$. The presence of the spin on the redox center turns on two of the three magnetic couplings, $J_{13}$ and $J_{23}$, which, in turn, influence the time evolution of the two-spin system. This fast, electrically controlled switching of the intramolecular magnetic interactions constitutes the essential ingredient of the quantum $\sqrt{\mathrm{SWAP}}$ gate detailed in refs 9,10 , and 28 , where two alternative read-out mechanisms are also proposed.

\section{CONCLUSIONS}

In summary, we show that incorporating an organic neutral diradical molecule in a three-terminal device allows for 
reversible and stable charging from the neutral state to its reduced state by means of a gate voltage. By performing IETS on both redox states, we find that the electron added onto the redox center magnetically couples to the radical spins, thereby driving the two-spin singlet into a three-spin doublet ground state (with three exchange couplings). In this way, by controlling the occupation of the redox center, the exchange interactions between the two radical spins and the added electron are switched on and off. Due to the large coupling to the leads, this switching takes place within subpicosecond time scales.

\section{METHODS}

Details on the Molecule. The studied molecule is a neutral 2,4,6trichloro- $\alpha, \alpha, \alpha^{\prime}, \alpha^{\prime}, \alpha^{\prime \prime}, \alpha^{\prime \prime}$-hexakis(pentachlorophenyl)mesitylene diradical prepared as previously reported. ${ }^{30}$ Electron spin resonance spectroscopy in frozen solutions containing the molecules shows an $S=1$ high-spin ground state, indicative of ferromagnetic exchange interactions between the two radical carbons in the molecule.

Junction Preparation. The molecular solution is prepared in a water-free glovebox environment. A small amount of molecular powder is dissolved in nitrogen-saturated dichlorobenzene to a concentration of approximately $0.5 \mathrm{mM}$.

The molecular solution is deposited by drop-casting onto a $\mathrm{Si} / \mathrm{SiO}_{2}$ chip containing several Au bridges $100 \mathrm{~nm}$ wide, $400 \mathrm{~nm}$ long, and 12 $\mathrm{nm}$ thick on top of a $\mathrm{AuPd} / \mathrm{Al}_{2} \mathrm{O}_{3}$ gate. The nanometer-spaced source-drain electrodes are produced by feedback-controlled electromigration of these bridges. ${ }^{31}$ The electromigration process is stopped when the bridge conductance reaches $3-4 G_{0}$. The wire is thereafter allowed to self-break at room temperature.

A total of 160 junctions were measured, 13 of which showed signatures characteristic of spin-dependent molecular transport. Eleven of these 13 showed clear singlet-triplet excitations with antiferromagnetic coupling ranging from 0.1 to about $11 \mathrm{meV}$; one showed a triplet-singlet characteristic with a ferromagnetic coupling of $2 \mathrm{meV}$. Four out of the 11 exhibited a degeneracy point and thus charging within the available gate voltage window. In three of the four, the added charge modulates the magnetic properties.

Experimental Conditions. All the measurements reported here are performed in a high-vacuum chamber $\left(P<5 \times 10^{4} \mathrm{mbar}\right)$ of a dilution refrigerator $(\sim 70 \mathrm{mK})$. A built-in superconducting magnet can be used to apply magnetic fields up to $9 \mathrm{~T}$.

Electrical current $I$ measurements are performed applying a dc bias voltage $V$ to the source and drain gold electrodes and/or a dc gate voltage $V_{\mathrm{g}}$ while recording $I$. The differential conductance $\mathrm{d} I / \mathrm{d} V$ is obtained by taking the numerical derivative of $I$.

\section{ASSOCIATED CONTENT}

\section{S Supporting Information}

The Supporting Information is available free of charge on the ACS Publications website at DOI: 10.1021/acsnano.7b01578.

Additional details about the fits of the magnetic excitation spectra and the DFT calculations (PDF)

\section{AUTHOR INFORMATION}

\section{Corresponding Author}

*E-mail: E.BurzuriLinares@tudelft.nl.

\section{ORCID}

Daniel Reta: 0000-0003-0000-9892

Jaume Veciana: 0000-0003-1023-9923

Enrique Burzurí: 0000-0001-7906-7192

\section{Present Address}

"School of Chemistry, The University of Manchester, Oxford Road, Manchester, M13 9PL United Kingdom.

\section{Notes}

The authors declare no competing financial interest.

\section{ACKNOWLEDGMENTS}

We acknowledge financial support by the Dutch Organization for Fundamental Research (NWO/FOM), an advanced ERC grant (Mols@Mols), and The Netherlands Organisation for Scientific Research (NWO/OCW) as part of the Frontiers of Nanoscience program. E.B. acknowledges funds from the EU FP7 program through Project 618082 ACMOL and a NWOVENI fellowship. C.R. and J.V. are thankful for funds from Networking Research Center on Bioengineering, Biomaterials, and Nanomedicine (CIBER-BBN), MINECO, Spain (CTQ2013-40480-R, CTQ 2016-80030-R, and "Severo Ochoa" Programme for Centers of Excellence in R\&D, SEV2015-0496), MCSA ITN Network i-Switch (GA 642196), and Generalitat de Catalunya (2014-SGR-17).

\section{REFERENCES}

(1) Wolf, S. A.; Awschalom, D. D.; Buhrman, R.; Daughton, J. M.; von Molnár, S.; Roukes, M. L.; Chtchelkanova, A. Y.; Treger, D. M. Spintronics: a Spin-Based Electronics Vision for the Future. Science 2001, 294, 1488-1495.

(2) Sanvito, S. Molecular Spintronics. Chem. Soc. Rev. 2011, 40, $3336-3355$

(3) Urdampilleta, M.; Klyatskaya, S.; Cleuziou, J.-P.; Ruben, M.; Wernsdorfer, W. Supramolecular Spin Valves. Nat. Mater. 2011, 10, 502-506.

(4) Bogani, L.; Wernsdorfer, W. Molecular Spintronics using SingleMolecule Magnets. Nat. Mater. 2008, 7, 179-186.

(5) Vincent, R; Klyatskaya, S.; Ruben, M.; Wernsdorfer, W.; Balestro, F. Electronic Read-Out of a Single Nuclear Spin using a Molecular Spin Transistor. Nature 2012, 488, 357-360.

(6) Thiele, S.; Balestro, F.; Ballou, R.; Klyatskaya, S.; Ruben, M.; Wernsdorfer, W. Electrically Driven Nuclear Spin Resonance in SingleMolecule Magnets. Science 2014, 344, 1135-1138.

(7) Lehmann, J.; Gaita-Ariño, A.; Coronado, E.; Loss, D. Quantum Computing with Molecular Spin Systems. J. Mater. Chem. 2009, 19, $1672-1677$

(8) Troiani, F.; Affronte, M.; Candini, A.; Ghirri, A.; Biagi, R.; del Pennino, U.; Carretta, S.; Garlatti, E.; Santini, P.; Amoretti, G.; Timco, G.; Winpenny, R. E. P.; Affronte, M.; Nakahara, M.; Hara, H.; Carl, P.; Höfer, P.; Takui, T. Molecular Spins for Quantum Information Technologies. Chem. Soc. Rev. 2011, 40, 3119-3129.

(9) Ferrando-Soria, J.; Magee, S. A.; Chiesa, A.; Carretta, S.; Santini, P.; Vitorica-Yrezabal, I. J.; Tuna, F.; Whitehead, G. F.; Sproules, S.; Lancaster, K. M.; Barra, A.-L.; Timco, G. A.; McInnes, E. J.; Winpenny, R. E. Switchable Interaction in Molecular Double Qubits. Chem. 2016, $1,727-752$.

(10) Ferrando-Soria, J.; Moreno Pineda, E.; Chiesa, A.; Fernandez, A.; Magee, S. A.; Carretta, S.; Santini, P.; Vitorica-Yrezabal, I. J.; Tuna, F.; Timco, G. A.; McInnes, E. J.; Winpenny, R. E. A Modular Design of Molecular Qubits to Implement Universal Quantum Gates. Nat. Commun. 2016, 7, 11377.

(11) Trif, M.; Troiani, F.; Stepanenko, D.; Loss, D. Spin-Electric Coupling in Molecular Magnets. Phys. Rev. Lett. 2008, 101, 217201.

(12) Osorio, E. A.; Moth-Poulsen, K.; van der Zant, H. S. J.; Paaske, J.; Hedegård, P.; Flensberg, K.; Bendix, J.; Bjørnholm, T. Electrical Manipulation of Spin States in a Single Electrostatically Gated Transition-Metal Complex. Nano Lett. 2010, 10, 105-110.

(13) Trif, M.; Troiani, F.; Stepanenko, D.; Loss, D. Spin Electric Effects in Molecular Antiferromagnets. Phys. Rev. B: Condens. Matter Mater. Phys. 2010, 82, 045429.

(14) Islam, M. F.; Nossa, J. F.; Canali, C. M.; Pederson, M. FirstPrinciples Study of Spin-Electric Coupling in a Cu3 Single Molecular Magnet. Phys. Rev. B: Condens. Matter Mater. Phys. 2010, 82, 155446. 
(15) Florens, S.; Freyn, A.; Roch, N.; Wernsdorfer, W.; Balestro, F.; Roura-Bas, P.; Aligia, A. A. Universal Transport Signatures in TwoElectron Molecular Quantum Dots: Gate-Tunable Hund's Rule, Underscreened Kondo Effect and Quantum Phase Transitions. J. Phys.: Condens. Matter 2011, 23, 243202.

(16) Palii, A.; Clemente-Juan, J. M.; Tsukerblat, B.; Coronado, E.; Glover, S. D.; Kubiak, C. P.; Long, J. R.; Rogez, G.; Yamada, T. K.; Ohresser, P.; Beaurepaire, E.; Wulfhekel, W. Electric Field Control of the Optical Properties in Magnetic Mixed-Valence Molecules. Chem. Sci. 2014, 5, 3598.

(17) Cardona-Serra, S.; Clemente-Juan, J. M.; Coronado, E.; GaitaAriño, A.; Suaud, N.; Svoboda, O.; Bastardis, R.; Guihéry, N.; Palacios, J. J. Electrically Switchable Magnetic Molecules: Inducing a Magnetic Coupling by Means of an External Electric Field in a Mixed-Valence Polyoxovanadate Cluster. Chem. - Eur. J. 2015, 21, 763-769.

(18) Burzurí, E.; Zyazin, A. S.; Cornia, A.; van der Zant, H. S. J. Direct Observation of Magnetic Anisotropy in an Individual Fe4 Single-Molecule Magnet. Phys. Rev. Lett. 2012, 109, 147203.

(19) Nossa, J. F.; Islam, M. F.; Canali, C. M.; Pederson, M. R. Electric Control of a $\mathrm{Fe} 4$ Single-Molecule Magnet in a Single-Electron Transistor. Phys. Rev. B: Condens. Matter Mater. Phys. 2013, 88, 224423.

(20) Misiorny, M.; Burzurí, E.; Gaudenzi, R.; Park, K.; Leijnse, M.; Wegewijs, M. R.; Paaske, J.; Cornia, A.; van der Zant, H. S. J. Probing Transverse Magnetic Anisotropy by Electronic Transport through a Single-Molecule Magnet. Phys. Rev. B: Condens. Matter Mater. Phys. 2015, 91, 035442.

(21) Scarrozza, M.; Barone, P.; Sessoli, R.; Picozzi, S.; Barry, M.; Doran, A.; Cruz, M. P.; Chu, Y. H.; Ederer, C.; Spaldin, N. A.; Das, R. R.; Kim, D. M.; Baek, S. H.; Eom, C. B.; Ramesh, R. Magnetoelectric Coupling and Spin-Induced Electrical Polarization in Metal-Organic Magnetic Chains. J. Mater. Chem. C 2016, 4, 4176-4185.

(22) Rajca, A.; Wongsriratanakul, J.; Rajca, S. Magnetic Ordering in an Organic Polymer. Science 2001, 294, 1503-1505.

(23) Li, T.; Tan, G.; Shao, D.; Li, J.; Zhang, Z.; Song, Y.; Sui, Y.; Chen, S.; Fang, Y.; Wang, X. Magnetic Bistability in a Discrete Organic Radical. J. Am. Chem. Soc. 2016, 138, 10092-10095.

(24) Mas-Torrent, M.; Crivillers, N.; Mugnaini, V.; Ratera, I.; Rovira, C.; Veciana, J.; Wöll, C.; Prato, S.; Pittana, P.; Manassen, Y.; Reinhoudt, D. N. Organic Radicals on Surfaces: Towards Molecular Spintronics. J. Mater. Chem. 2009, 19, 1691-1695.

(25) Frisenda, R.; Gaudenzi, R.; Franco, C.; Mas-Torrent, M.; Rovira, C.; Veciana, J.; Alcon, I.; Bromley, S. T.; Burzurí, E.; van der Zant, H. S. J. Kondo Effect in a Neutral and Stable All Organic Radical Single Molecule Break Junction. Nano Lett. 2015, 15, 3109-3114.

(26) Gaudenzi, R.; Burzurí, E.; Reta, D.; Moreira, I. d. P. R.; Bromley, S. T.; Rovira, C.; Veciana, J.; van der Zant, H. S. J. Exchange Coupling Inversion in a High-Spin Organic Triradical Molecule. Nano Lett. 2016, 16, 2066-2071.

(27) Simão, C.; Mas-Torrent, M.; Crivillers, N.; Lloveras, V.; Artés, J. M.; Gorostiza, P.; Veciana, J.; Rovira, C. A Robust Molecular Platform for Non-Volatile Memory Devices with Optical and Magnetic Responses. Nat. Chem. 2011, 3, 359-364.

(28) Lehmann, J.; Gaita-Ariño, A.; Coronado, E.; Loss, D. Spin Qubits with Electrically Gated Polyoxometalate Molecules. Nat. Nanotechnol. 2007, 2, 312-317.

(29) Luis, F.; Repollés, A.; Martínez-Pérez, M. J.; Aguilá, D.; Roubeau, O.; Zueco, D.; Alonso, P. J.; Evangelisti, M.; Camón, A.; Sesé, J.; Barrios, L. A.; Aromí, G. Molecular Prototypes for Spin-Based CNOT and SWAP Quantum Gates. Phys. Rev. Lett. 2011, 107, 117203.

(30) Veciana, J.; Rovira, C.; Ventosa, N.; Crespo, M. I.; Palacio, F. Stable Polyradicals with High-Spin Ground States. 2. Synthesis and Characterization of a Complete Series of Polyradicals Derived from 2,4,6-Trichloro-.alpha.,.alpha.,.alpha., ,.alpha., ,.alpha.",.alpha."-hexakis(pentachlorophenyl)mesitylene with $S=1 / 2$. J. Am. Chem. Soc. 1993, $115,57-64$.

(31) Burzurí, E.; Gaudenzi, R.; van der Zant, H. S. J. Observing Magnetic Anisotropy in Electronic Transport through Individual Single-Molecule Magnets. J. Phys.: Condens. Matter 2015, 27, 113202.
(32) Perrin, M. L.; Burzurí, E.; van der Zant, H. S. J. Single-Molecule Transistors. Chem. Soc. Rev. 2015, 44, 902-919.

(33) Ternes, M. Spin Excitations and Correlations in Scanning Tunneling Spectroscopy. New J. Phys. 2015, 17, 063016.

(34) Kondo, J. Resistance Minimum in Dilute Magnetic Alloys. Prog. Theor. Phys. 1964, 32, 37-49.

(35) Liang, W.; Shores, M. P.; Bockrath, M.; Long, J. R.; Park, H. Kondo Resonance in a Single-Molecule Transistor. Nature 2002, 417, 725-729.

(36) Souto, M.; Lloveras, V.; Vela, S.; Fumanal, M.; Ratera, I.; Veciana, J. Three Redox States of a Diradical Acceptor-DonorAcceptor Triad: Gating the Magnetic Coupling and the Electron Delocalization. J. Phys. Chem. Lett. 2016, 7, 2234-2239. 